Article

\title{
Hysteretic Loops in Correlation with the Maximum Dissipated Energy, for Linear Dynamic Systems
}

\author{
Polidor Bratu
}

Research Institute for Construction Equipment and Technology-ICECON S.A., 021652 București, Romania; icecon@icecon.ro; Tel.: +40-021-202-5500

Received: 17 December 2018; Accepted: 21 February 2019; Published: 2 March 2019

\begin{abstract}
This paper presents the outcomes of the theoretical and experimental research carried out on a real model at natural scale using Voigt-Kelvin linear viscoelastic type $m, c$, and $k$ models excited by a harmonic force $F(t)=F_{0} \sin \omega t$, where $F_{0}$ is the amplitude of the harmonic force and $\omega$ is the excitation angular frequency. The linear viscous-elastic rheological system $(m, c, k)$ is characterized by the fact that the $c$ linear viscous damping-and, consequently, the fraction of the critical damping $\zeta$-may be changed so that the dissipated energy can reach maximum $W_{d}^{\max }$ values. The optimization condition between the $W_{d}^{\max }$ maximum dissipated energy and the amortization $\zeta^{0}= \pm\left(1-\Omega^{2}\right) / 2 \Omega$ modifies the structure of the relation $F=F(x)$, which describes the elliptical hysteresis loop $F-x$ in the sense that it has its large axis making an angle less than $90^{\circ}$ with respect to the $\mathrm{x}$-axis in $\Omega<1$ ante-resonance, and an angle greater than $90^{\circ}$ in post-resonance for $\Omega>1$. The elliptical $Q-x$ hysteretic loops are tilted with their large axis only at angles below $90^{\circ}$. It can be noticed that the equality between the arias of the hysteretic loop, in the two representations systems $Q-x$ and $F-x$, is verified, both being equal with the maximum dissipated energy $W_{d}^{\max }$.
\end{abstract}

Keywords: hysteretic loops; dissipated energy; linear dynamic systems

\section{Introduction}

For the vibration-driven technological processes with excitation harmonic forces, Voigt-Kelvin linear rheological models are adopted with the discretionary variation of the amortization or of the fraction of the critical amortization $\zeta=c / 2 \sqrt{\mathrm{k} / \mathrm{m}}$.

The functional optimization of the vibration-driven processes consists of reaching a maximum $W_{d}^{\max }$ dissipated energy for $\zeta=\zeta^{0}$ in direct correlation with the area of the elliptical hysteretic loop. By locating suitable transducers on the physical system, the elliptical hysteretic loops can be determined in such a manner that allows the assessment of their areas, respectively the maximum dissipated energy for a dynamic system may be assessed with rigidity $k$ and amplitude $A$ of the instantaneous displacement $x=A \sin (\omega t-\varphi)$, where $\varphi$ is the phase angle.

Depending on the optimization requirement, in order to achieve a maximum dissipation, i.e., $W_{d}^{\max }$, can be determined the pairs of values $\zeta_{\text {optim }}=\zeta^{0}$ for which are established the calculus relations of the significant parameters. Thus, the functional relations were established for $A=A(\omega)$, amplitude, the $W_{d}=W_{d}(\zeta, \Omega)$ dissipated energy, the viscoelastic force $Q=c \dot{x}+k x=Q(x)$, and the excitation force $F=F(x)$. The correlations between the maximum dissipated energy and the areas of the elliptical hysteretic loops are reflected in the graphical representations for the case of operation in ante-resonance at $\Omega<1$ and respectively for the case of post-resonance at $\Omega>1$ [1-3].

Experimental research carried out so far has mainly highlighted the positioning of hysteretic loops as an ante-resonance regime for testing elastomeric devices. The results highlighted in this field, both linearly and nonlinearly, are presented in papers published by various researchers from Italy, France, USA, and Romania [4-10]. Interpretation of experimental results and behavioral modeling 
of elastomeric device behavior are addressed in published reference works [11-18]. To highlight the post-resonance regime, interesting and useful research is contained in some published papers cited in the literature [19-23].

In this paper are highlighted the hysteretic loops families of the elastomeric devices that were designed, manufactured and tested, for a viaduct on the "Transilvania" Motorway, in Romania. Thus, based on dynamic testing devices and realized within ICECON Bucharest (Romania), there were determined the characeristic shapes of the hysteretic loops for the three dynamic regimmes, namely: ante-resonance, resonance, and post-resonance.

The characteristic function for dissipated energy was also established, highlighting its modification in relation to the fraction of critical damping $\zeta$ as a system parameter.

The novelty of the approach consists of the fact that for the elastomeric isolator system/device are highlighted the dissipative characteristics, the hysteretic loop orientation, as well as the dissipated energy variation depending on the parameter $\zeta$, which can take different values in relation to mass $m$, elasticity $k$, and linear dissipation $c$. The graphical representation of the hysteretic loop in accordance with the dissipated energy allowed a clearer, more operational and efficient highlighting of the dynamic behavior during the tests, with meaningful and eloquent conclusions.

\section{Parameter Analysis}

The dynamic linear models $m, c$, and $k$, produced by the harmonic force $F(t)=F_{0} \sin \omega t$ and with the reaction force $Q(t)$ in the liniar viscoelastic system, $c, k$, is presented in Figure 1.

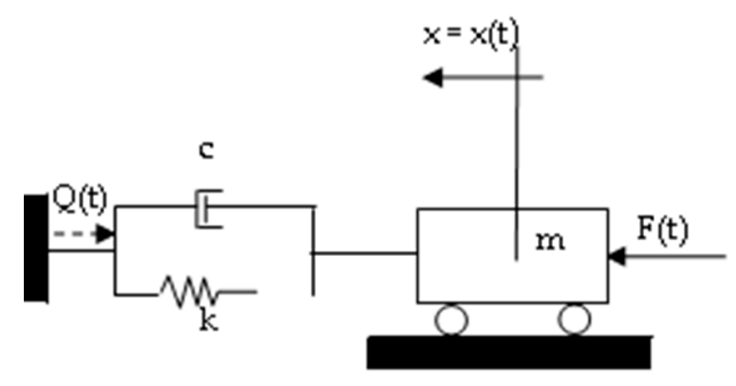

Figure 1. Linear dynamic model $m, c$, and $k$.

The movement equation at the dynamic balance is

$$
m \ddot{x}+c \dot{x}+k x=F_{0} \sin \omega t .
$$

The solution of Equation (1) is

$$
x=A \sin (\omega t-\varphi),
$$

where $A$ is the amplitude of the instantaneous displacement $x=x(t), \varphi$ is the phase shift between the instantaneous displacement $x(t)$ and the excitation force $F(t)$.

It is introduced the notation: $\Omega=\frac{\omega}{\omega_{n}}$, where $\omega_{n}=\sqrt{\frac{k}{m}}$, so that the variable $\Omega$ to be used as excitation relative pulsation.

\subsection{Amplitude of Displacement}

An amplitude of the instantaneous displacement $x=x(t)$ may be expressed by the $A(\omega)$ or $A(\Omega)$ relation, as follows:

$$
A(\omega)=\frac{F_{0}}{\sqrt{\left(k-m \omega^{2}\right)^{2}+c^{2} \omega^{2}}}
$$


or

$$
A(\omega)=\frac{F_{0}}{k} \frac{1}{\sqrt{\left(1-\Omega^{2}\right)^{2}+(2 \zeta \Omega)^{2}}} .
$$

\subsection{De-Phasing between Force and Displacement}

The de-phasing $\varphi$ between the excitation force $F=F(t)$ and the instantaneous displacement $x=$ $x(t)$ may be calculated as follows:

$$
\left.\operatorname{tg} \varphi\right|_{\omega}=\frac{c \omega}{k-m \omega^{2}}
$$

or

$$
\left.\operatorname{tg} \varphi\right|_{\Omega}=\frac{2 \zeta \Omega}{1-\Omega^{2}}
$$

\subsection{Dissipated Energy}

The dissipated energy $W_{d}$ may be expressed according to $\Omega$ and $\zeta$ as follows:

$$
W_{d}=W_{d}(\Omega, \zeta)=W_{0} \frac{2 \zeta \Omega}{\left(1-\Omega^{2}\right)^{2}+(2 \zeta \Omega)^{2}},
$$

where $W_{0}=\pi \frac{F_{0}}{k}$.

From the condition of maximum $W_{d}$, which is $\frac{d W_{d}}{d \zeta}=0$, it emerges that $\zeta^{0}= \pm \frac{1-\Omega^{2}}{2 \Omega}$, so that the maximum dissipated energy $W_{d}^{\max }$ becomes

$$
W_{d}^{\max }=W_{0} \frac{1}{2\left(1-\Omega^{2}\right)}=W_{0} \frac{1}{2(2 \zeta \Omega)} .
$$

It can be noticed that for certain values of $\Omega$ it correspond the values of $\zeta^{0}$ for which the dissipated energy is at maximum. The representation of $W_{d}(\zeta)$, so that the variation domain of $\zeta$ to be included $\zeta^{0}$, leads to a curve with a maximum point, having the coordinates $\zeta^{0}$ at $W_{d}^{\max }$. For the discrete values of $\Omega$, a family of parametrical curves of $W_{d}(\Omega, \zeta)$ can be obtained.

\subsection{Equation of the F-x Elliptic Hysteretic Loop}

Phase $\omega t$ is eliminated between $x=x(t)$ and $F=F(t)$ as follows:

$$
\left\{\begin{array}{c}
x=A \sin \omega t \cos \varphi-A \sin \varphi \cos \omega t \\
F=F_{0} \sin \omega t
\end{array},\right.
$$

where $\sin \omega t=\frac{F}{F_{0}}, \cos \omega t= \pm \sqrt{1-\frac{F^{2}}{F_{0}^{2}}}$, resulting

$$
\frac{x}{A}=\frac{F}{F_{0}} \cos \varphi \mp \sin \varphi \sqrt{1-\frac{F^{2}}{F_{0}^{2}}} .
$$

If we note the dimensionless sizes $\frac{x}{A}=X$ and $\frac{F}{F_{0}}=\Phi$, then relation (10) becomes

$$
X=\Phi \cos \varphi \mp \sin \varphi \sqrt{1-\Phi^{2}} .
$$

From of equation (11) $\Phi$ emerges as

$$
\Phi=X \cos \varphi \pm \sin \varphi \sqrt{1-X^{2}},
$$


where $\sin \varphi=\frac{c \omega}{\sqrt{\left(k-m \omega^{2}\right)^{2}+c^{2} \omega^{2}}}$ and $\cos \varphi=\frac{k-m \omega^{2}}{\sqrt{\left(k-m \omega^{2}\right)^{2}+c^{2} \omega^{2}}}$.

By replacing $\frac{x}{A}=X, \frac{F}{F_{0}}=\Phi$, $\sin \varphi$, and $\cos \varphi$ in relation (12), the expression of force $F$ emerges according to pulsation $\omega$, rigidity $k$, and amortization $c$ as follows:

$$
F(x)=F(x, \omega)=\left(k-m \omega^{2}\right) x \pm c \omega \sqrt{A^{2}(\omega)-x^{2}},
$$

or in terms of $\Omega$ and $\zeta$ measures we have

$$
F(x, \Omega)=k\left[\left(1-\Omega^{2}\right) x \pm 2 \zeta \Omega \sqrt{A^{2}(\Omega)-x^{2}}\right] .
$$

For the condition that the dissipated energy to be maximum, $W_{d}=W_{d}^{\max }$ at $\zeta_{\text {optim }}=\zeta^{0}$.

(a) For the ante-resonance regime $\Omega<1$ at $\zeta_{a}^{0}=\frac{1-\Omega^{2}}{2 \Omega}$, obtaining

$$
F(x, \Omega)=\left(1-\Omega^{2}\right) k\left[x \pm \sqrt{A^{2}(\Omega)-x^{2}}\right],
$$

or

$$
F(x, \zeta, \Omega)=2 \zeta_{a}^{0} \Omega k\left[x \pm \sqrt{A^{2}\left(\Omega, \zeta_{a}^{0}\right)-x^{2}}\right] .
$$

(b) For the post-resonance regime $\Omega>1$ at $\zeta_{p}^{0}=-\frac{1-\Omega^{2}}{2 \Omega}$ the following is obtained:

$$
F(x, \Omega)=\left(1-\Omega^{2}\right) k\left[x \pm \sqrt{A^{2}(\Omega)-x^{2}}\right],
$$

or

$$
F(x, \zeta, \Omega)=-2 \zeta_{p}^{0} \Omega k\left[x \pm \sqrt{A^{2}\left(\Omega, \zeta_{p}^{0}\right)-x^{2}}\right]
$$

where

$$
A(\Omega)= \pm \frac{F_{0}}{k} \frac{1}{\left(1-\Omega^{2}\right) \sqrt{2}}
$$

or

$$
A\left(\Omega, \zeta_{a, p}^{0}\right)= \pm \frac{F_{0}}{k} \frac{1}{2 \zeta_{a, p}^{0} \Omega \sqrt{2}}
$$

\subsection{Equation of the Hysteretic Loop $Q-x$}

The instantaneous viscous-elastic force $Q(t)=c \dot{x}+k x$ may be expressed from the dynamic equilibrium equation. Thus, it can be expressed $m \ddot{x}+c \dot{x}+k x=F(t)$ or

$$
m \ddot{x}+Q(t)=F(t)
$$

from where $Q(t)$ emerges as $Q(t)=F(t)-m \ddot{x}$, or in relation with $x=x(t)$, it is obtained

$$
Q(x)=F(x)-m\left(-\omega^{2} x\right) .
$$

In Equation (22) we insert the expression of force $F(x)=F(x, \omega)$ from (13) and have

$$
Q(x)=Q(x, \omega)=k x \pm c \omega \sqrt{A^{2}(\omega)-x^{2}},
$$


and in relation with $\Omega$ Equation (23) becomes

$$
Q(x)=Q(x, \omega)=k\left[x \pm 2 \zeta \Omega \sqrt{A^{2}(\Omega)-x^{2}}\right] .
$$

It is found that the family of ellipses according to Equation (24) is characterized by the fact that all ellipses have their major axis in the quadrant $(0, \pi / 2)$ with positive angular coefficient $\operatorname{tg} \alpha=k>0$ for any value $\Omega>0$, where $\alpha$ is the angle between the ellipse axis and the $O x$ axis [24].

\section{Elliptic Hysteretic Loops for the Ante-Resonance Regime $(\Omega<1)$}

For a dynamic system where $m=10^{4} \mathrm{~kg}, k=2 \cdot 10^{7} \mathrm{~N} / \mathrm{m}$ at the relative pulses $\Omega_{1}=0.8$ with $\zeta_{\text {optim }}=\zeta_{1}^{0}=0.225 ; \Omega_{2}=0.6$ with $\zeta_{\text {optim }}=\zeta_{2}^{0}=0.53$; and $\Omega_{3}=0.5$ with $\zeta_{\text {optim }}=\zeta_{3}^{0}=0.75$, the representation of the elliptical hysteretic loops $Q-x$ and $F-x$, according to the graph of function $W_{d}=W(\zeta)$, as shown in Figures 2-4. In each figure functions $F(x)$ and $Q(x)$ are represented based on Equations (16) and (24), respectively, corresponding to the representation of function $W_{d}{ }^{\max }=W(\zeta)$, given by Equation (8). Thus, it is found that for the maximum value of the dissipated energy $W_{d}$ max according to $\Omega_{i}<1$ and $\zeta_{\text {optim }}=\zeta_{i}^{0}, i=1,2,3$, the areas of the ellipses $F-x$ and $Q-x$ are maximum and equal [25-29]

We specify the fact that the fraction of the critical amortization $\zeta_{e f}$, in this case for $W_{d}$ max , may be defined as follows:

$$
\zeta_{\text {ef }}=\frac{W_{d}^{\max }}{4 \pi \frac{1}{2} k A^{2}\left(\omega, \zeta^{0}\right)^{\prime}}
$$

and taking into account Equations (8) and (20), we have

$$
\zeta_{\text {ef }}=\zeta^{0} \Omega \text {. }
$$

It can be observed that only at a resonance, when $\Omega_{i}=1$, it results $\zeta_{\text {ef }}=\zeta^{0}$.

In Figures 2-5, the functions $W_{i}=W_{i}(\zeta), F_{i}-x$, and $Q_{i}-x$ are represented for $\zeta_{\text {optim }}=\zeta_{i}^{0}$ in which $W_{i}=W_{d}{ }^{\max }\left(\zeta^{0}\right)$ and $\Omega_{i}$ where $i=1,2,3$.

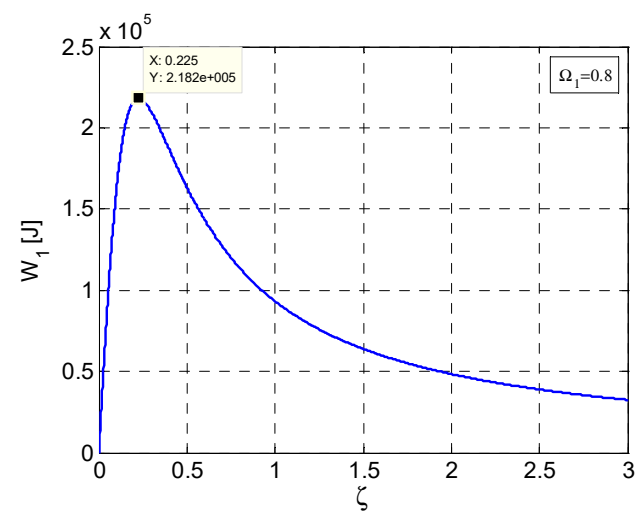

$(\mathbf{a}): \mathrm{W}_{1 \max }=2 \cdot 1 \cdot 10^{5} \mathrm{~J}$

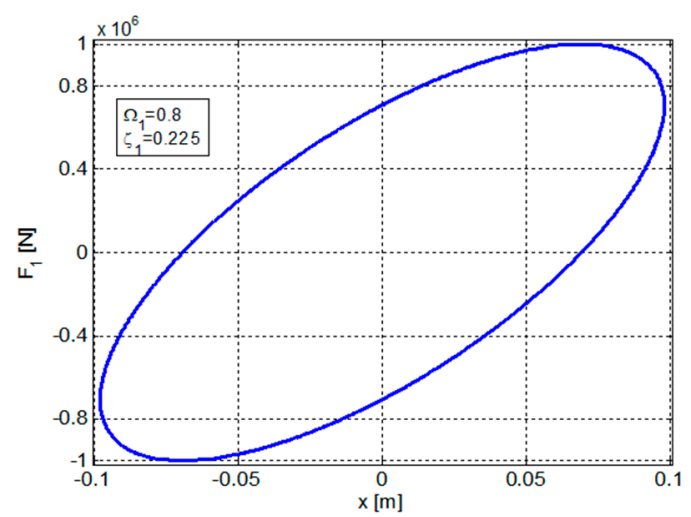

(b): area $=2 \cdot 1 \cdot 10^{5} \mathrm{~J}$

Figure 2. Cont. 


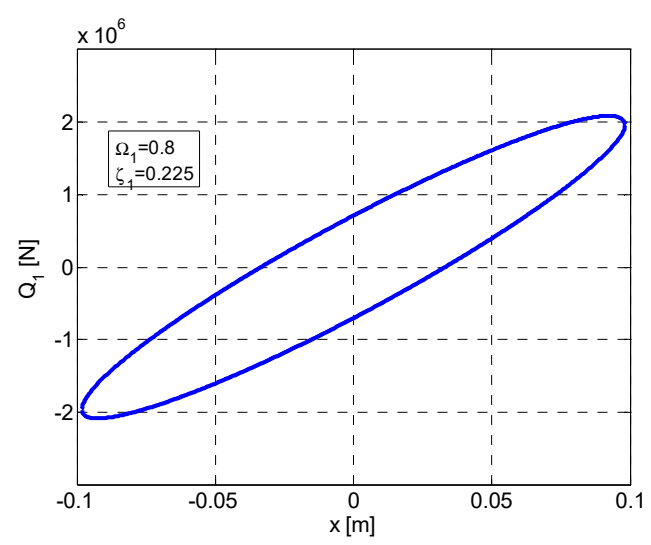

(c): area $=2 \cdot 1 \cdot 10^{5} \mathrm{~J}$

Figure 2. Variation curves in ante-resonance for $W_{1}{ }^{\text {max }}$ at $\Omega_{1}=0.8$ and $\zeta_{1}=0.225$. (a) Variation of $W_{1}$ according to $\zeta$. (b) Elliptic hysteretic loop $F_{1}-x$. (c) Elliptic hysteretic loop $Q_{1}-x$. $x=[-0.08701 \div$ $+0.08701]$.

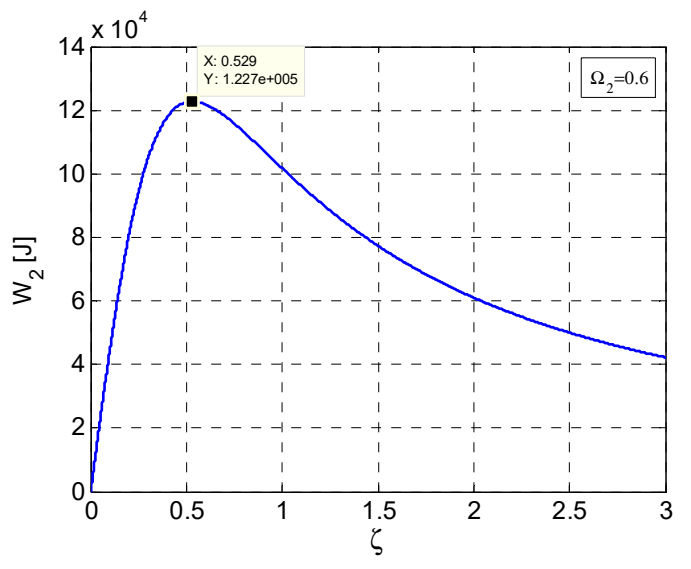

(a): $\mathrm{W}_{2 \max }=1.2 \cdot 10^{5} \mathrm{~J}$

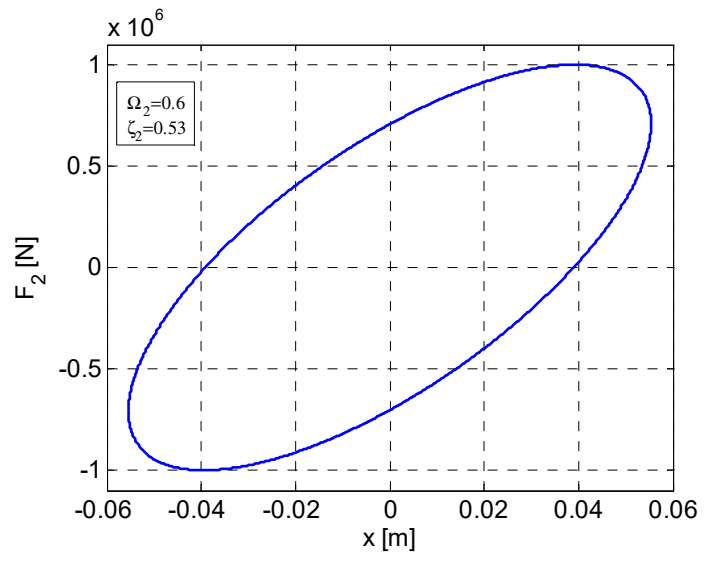

(b): area $=1.2 \cdot 10^{5} \mathrm{~J}$

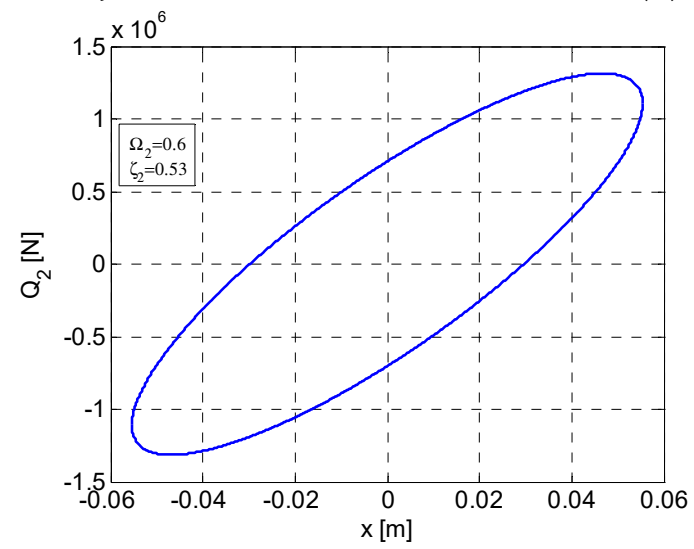

(c): area $=1.2 \cdot 10^{5} \mathrm{~J}$

Figure 3. Variation curves in ante-resonance for $W_{2}{ }^{\text {max }}$ at $\Omega_{2}=0.6$ and $\zeta_{2}=0.53$. (a) Variation of $W_{2}$ according to $\zeta$. (b) Elliptic hysteretic loop $F_{2}-x$. (c) Elliptic hysteretic loop $Q_{2}-x . x=[-0.05542 \div$ $+0.05542]$. 


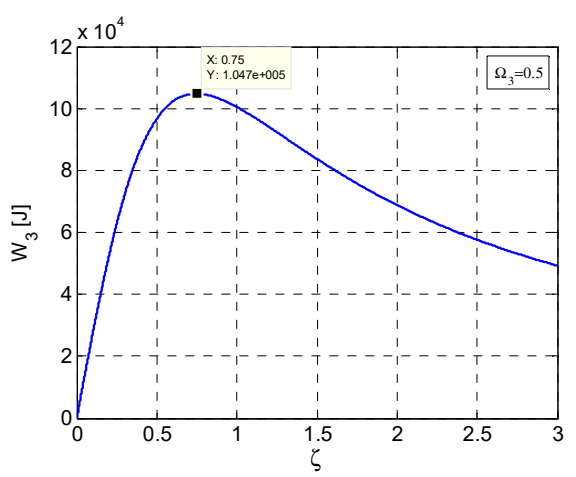

(a): W3 $\max =1.04 \cdot 105 \mathrm{~J}$

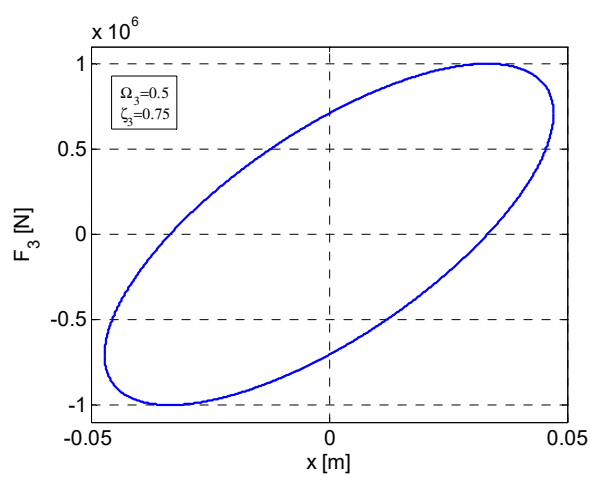

(b): area $=1.04 \cdot 105 \mathrm{~J}$

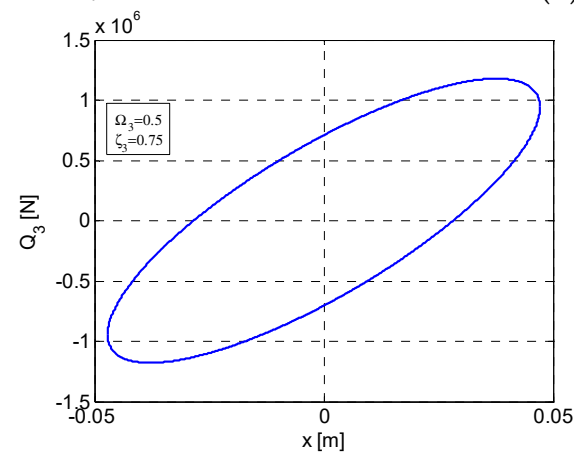

(c): area $=1.04 \cdot 105 \mathrm{~J}$

Figure 4. Variation curves in ante-resonance for $W_{3}^{\text {max }}$ at $\Omega_{3}=0.5$ and $\zeta_{3}=0.75$. (a) Variation of $W_{3}$ according to $\zeta$. (b) Elliptic hysteretic loop $F_{3}-x$. (c) Elliptic hysteretic loop $Q_{3}-x$. $x=[-0.04715 \div$ $+0.04715]$.

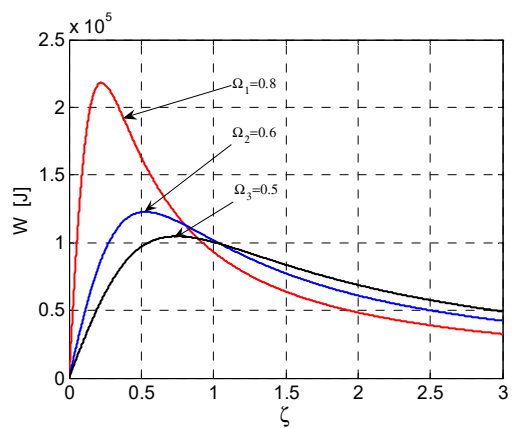

(a)

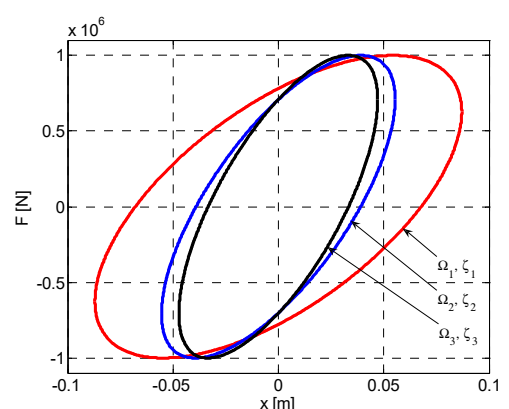

(b)

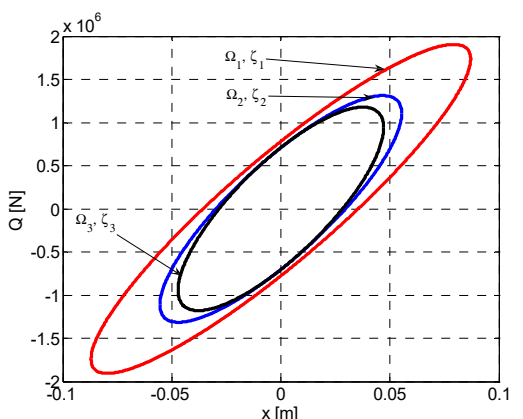

(c)

Figure 5. Families of curves in ante-resonance for $W_{1}^{\max }, W_{2}{ }^{\max }$, and $W_{3}{ }^{\max }$. (a) Families of curves $W$ according to the current variable $\zeta$ and the discreet variable $\Omega$. (b) The family of elliptical hysteretic loops $F-x$ according to the current variable $\mathrm{x}$ and the pair of discreet variables $\Omega, \zeta$. (c) The family of elliptical hysteretic loops $Q-x$ according to the current variable $\mathrm{x}$ and the pair of discreet variables $\Omega, \zeta$. 


\section{Elliptic Hysteretic Loops for the Post-Resonance Regime $(\Omega>1)$}

In the case of the above described system, for $\Omega_{4}=1.31$ with $\zeta_{4}^{0}=0.28 ; \Omega_{5}=1.66$ with $\zeta_{5}^{0}=0.53$; $\Omega_{6}=2$ with $\zeta_{6}^{0}=0.75$, and $\Omega_{7}=2.32$ with $\zeta_{7}^{0}=0.95$, in the Figures 6-10 there were represented the functions $W_{i}(\zeta)$ and $Q_{i}(x)$ were $i=4,5,6,7$.

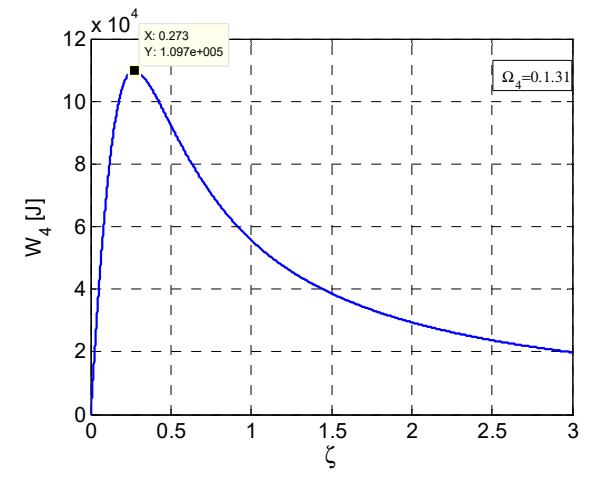

(a): $\mathrm{W}_{4 \max }=1.09 \cdot 10^{5} \mathrm{~J}$

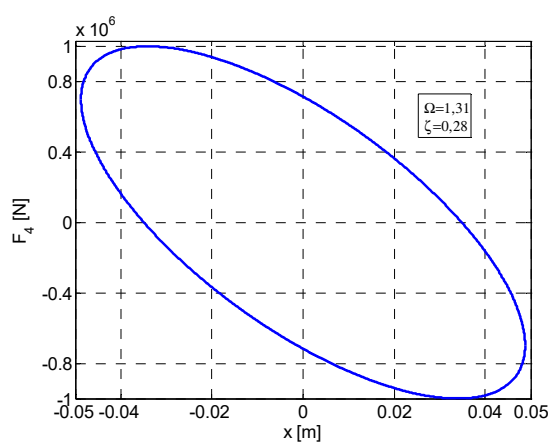

(b): area $=1.09 \cdot 10^{5} \mathrm{~J}$

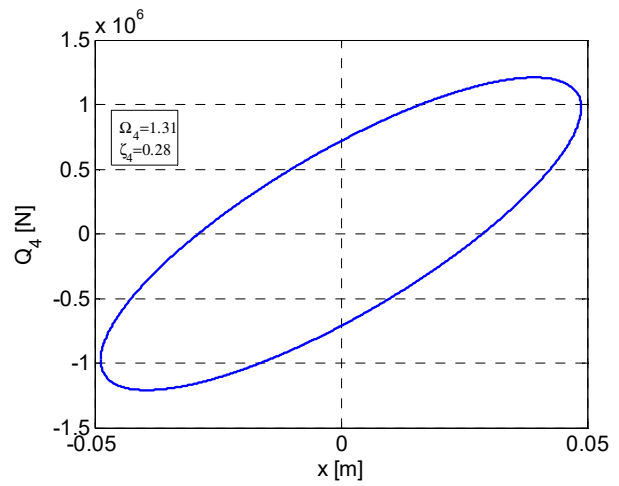

(c): area $=1.09 \cdot 10^{5} \mathrm{~J}$

Figure 6. Variation curves in post-resonance for $W_{4}{ }^{\text {max }}$ at $\Omega_{4}=1.31$ and $\zeta_{4}=0.28$. (a) Variation of $W_{4}$ according to $\zeta$. (b) Elliptic hysteretic loop $F_{4}-x$. (c) Elliptic hysteretic loop $Q_{4}-x$. $x=[-0.04878 \div$ $+0.04878]$.

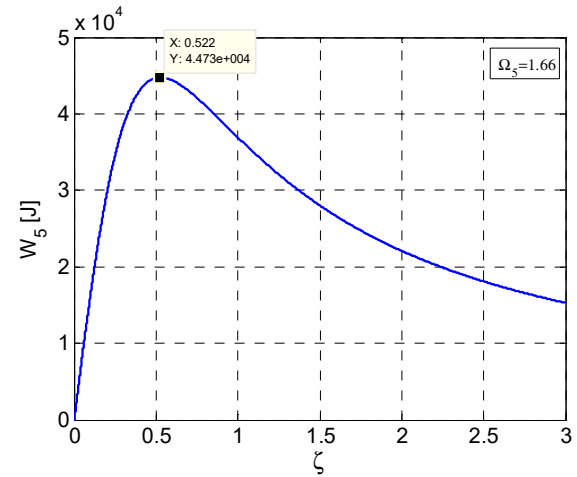

(a): $\mathrm{W}_{5 \max }=4.47 \cdot 10^{4} \mathrm{~J}$

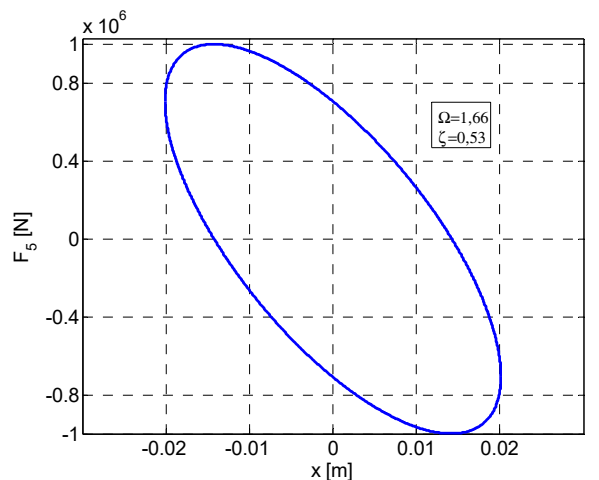

(b): area $=4.47 \cdot 10^{4} \mathrm{~J}$

Figure 7. Cont. 


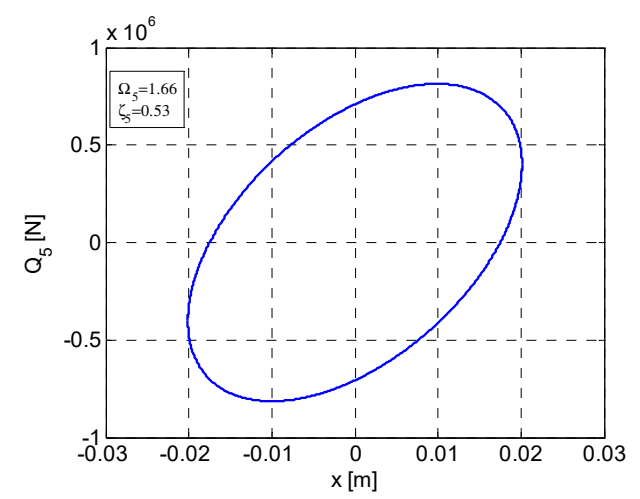

(c): area $=4.47 \cdot 10^{4} \mathrm{~J}$

Figure 7. Variation curves in post-resonance for $W_{5}{ }^{\text {max }}$ at $\Omega_{5}=1.66$ and $\zeta_{5}=0.53$. (a) Variation of $W_{5}$ according to $\zeta$. (b) Elliptic hysteretic loop $F_{5}-x$. (c) Elliptic hysteretic loop $Q_{5}-x$. $x=[-0.02012 \div$ $+0.02012]$.

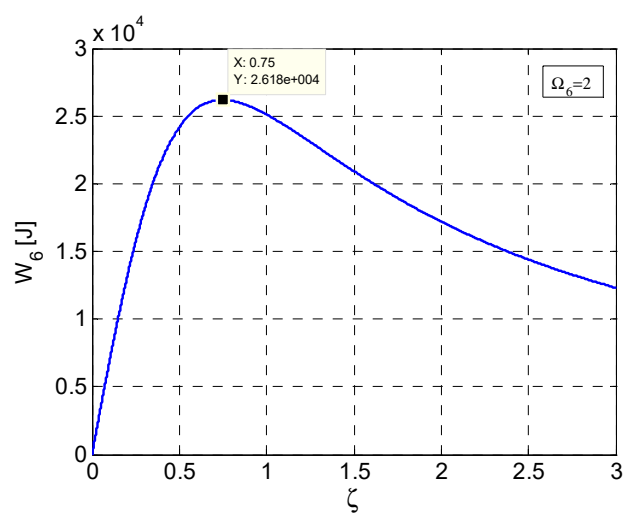

(a): $\mathrm{W}_{6 \max }=2.61 \cdot 10^{4} \mathrm{~J}$

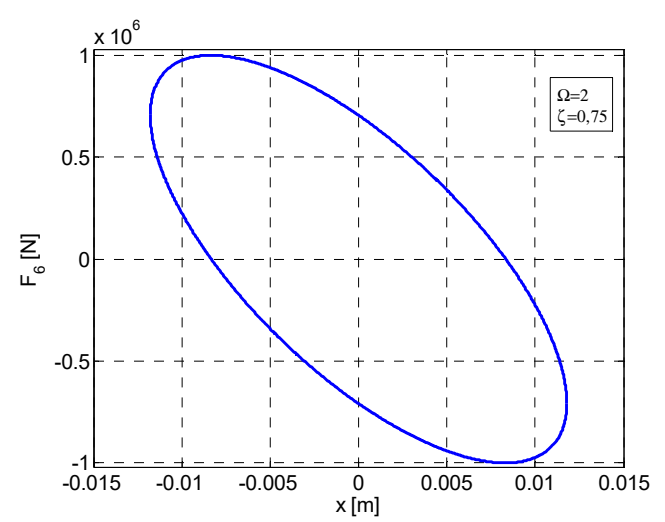

(b): area $=2.61 \cdot 10^{4} \mathrm{~J}$

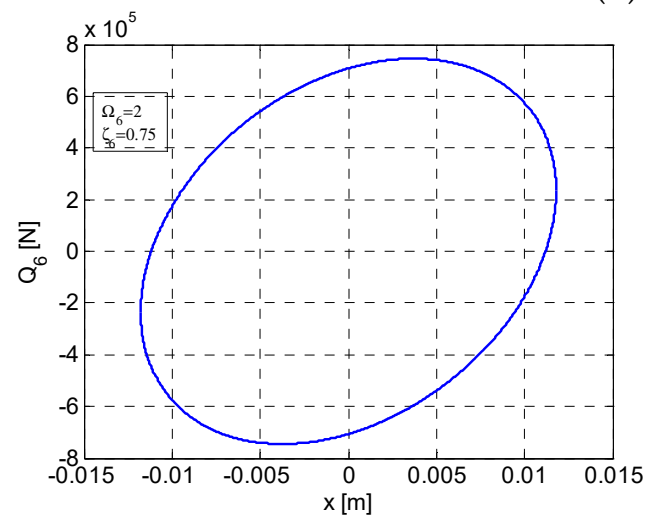

(c): area $=2.61 \cdot 10^{4} \mathrm{~J}$

Figure 8. Variation curves in post-resonance for $W_{6}{ }^{\max }$ at $\Omega_{6}=2$ and $\zeta_{6}=0.75$. (a) Variation of $W_{6}$ according to $\zeta$. (b) Elliptic hysteretic loop $F_{6}-x$. (c) Elliptic hysteretic loop $Q_{6}-x . x=[-0.01179 \div$ $+0.01179]$. 


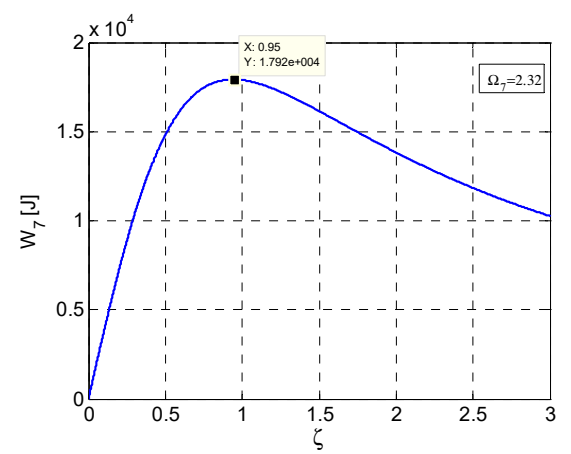

(a): $\mathrm{W}_{7 \max }=1.79 \cdot 10^{4} \mathrm{~J}$

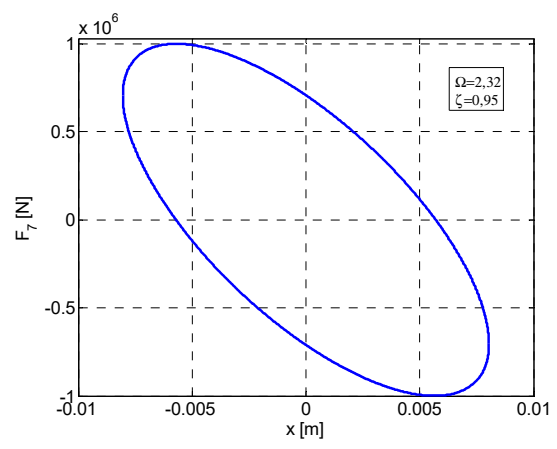

(b): area $=1.79 \cdot 10^{4} \mathrm{~J}$

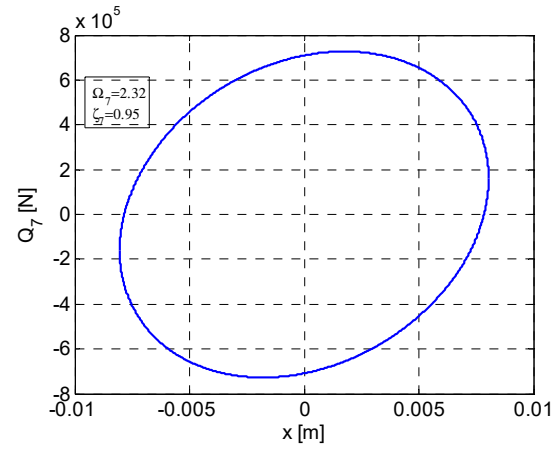

$(\mathbf{c})$ : area $=1.79 \cdot 10^{4} \mathrm{~J}$

Figure 9. Variation curves in post-resonance for $W_{7}{ }^{\max }$ at $\Omega_{7}=2.32$ and $\zeta_{7}=0.95$. (a) Variation of $W_{7}$ according to $\zeta$. (b) Elliptic hysteretic loop $F_{7}-x$. (c) Elliptic hysteretic loop $Q_{7}-x . x=[-0.008045 \div$ $+0.008045]$.

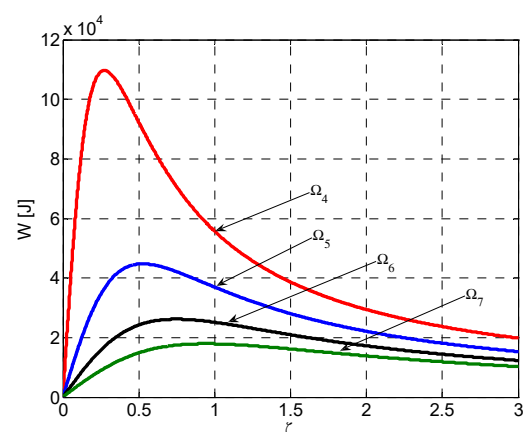

(a)

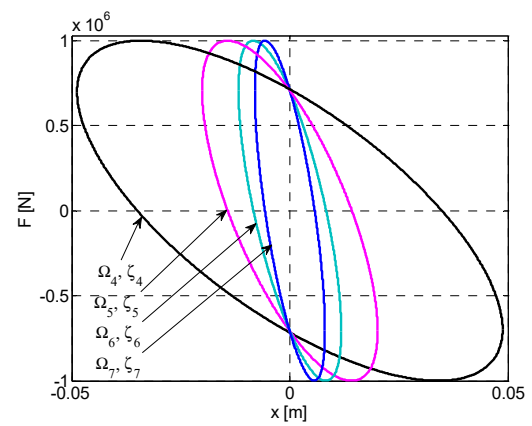

(b)

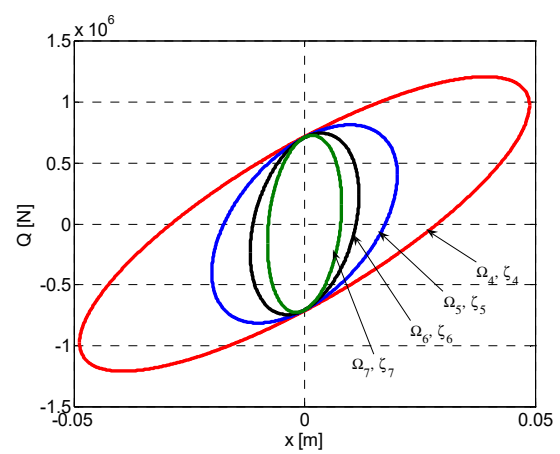

(c)

Figure 10. Families of curves in post-resonance for the maximum values of the dissipated energy $W_{4}, W_{5}, W_{6}$, and $W_{7}$ at parameters $\left(\Omega_{4}, \zeta_{4}\right),\left(\Omega_{5}, \zeta_{5}\right),\left(\Omega_{6}, \zeta_{6}\right)$, and $\left(\Omega_{7}, \zeta_{7}\right)$. (a) Families of curves $W$ parametrized by the discreet variable $\Omega$. (b) The family of elliptical hysteretic loops $F-x$ for the pair of variables $\Omega, \zeta$. (c) The family of elliptical hysteretic loops $Q-x$ for the pair of variables $\Omega, \zeta$. 


\section{Conclusions}

The dynamic linear system $m, c$, and $k$ excited by a harmonic perturbatory force is assessed dynamic regimes of ante-resonance $\Omega<1$ and and post-resonance $\Omega>1$ for values of the viscous dumping $\zeta=\zeta_{\text {optim }}=\zeta^{0}$, for which the dissipated energy is maximum: $W_{d}=W^{\max }\left(\zeta^{0}\right)$.

(a) For this, there are established the functional relations of the perturbatory force $F=F(x)$ in relation with the instantaneous displacement $x=x(t)$, of the viscoelastic force $Q=Q(t)$ in relation with $x=$ $x(t)$, of the viscoelastic force $Q=Q(x)$ in relation with $x=x(t)$, and of the dissipated energy in relation with the variation of the viscous dumping $\zeta$, namely $W_{d}=W(\zeta)$;

(b) In this context, based on the graphical representations, it was possible to emphasize the fact that the ellipses $F-x$ are rotating in relation to the origin of the axis system, according to the dynamic regime $\Omega<1$ or $\Omega>1$. The axis of the ellipse $F-x$ rotation is caused by the inertial effect that is produced by the presence of the factor $1-\Omega^{2}$ in the expression of the function $F=F(x)$;

(c) The representation of the function $Q=Q(x)$ highlights the that it constantly maintains an angle of inclination of the ellipse $Q-x$, axis, with a positive slope;

(d) It can be noticed that the areas of the hysteretic loops $F-x$ and $Q-x$ are equal between them and equal with the maximum value of the dissipated energy $W^{\max }\left(\zeta^{0}\right)$ for $\zeta^{0}=\frac{1-\Omega^{2}}{2 \Omega}$.

(e) The effective critical dumping $\zeta_{\text {ef }}$ depends both on the value of $\zeta^{0}$ as well as on the relative pulsation $\Omega<1$, for ante-resonance or $\Omega>1$, for post-resonance. In the case of the resonance regime, for $\Omega=1$, then $\zeta_{e f}=\zeta^{0}$ is a sole situation that enables the assessment of the fraction from the optimal critical amortization $\zeta^{0}$.

The concordant graphical representations eloquently demonstrated the fact that for the dynamic regimes defined by the relative pulsation $\Omega$, the dissipative effects of the linear viscous-elastic systems may be analyzed, in experimental conditions defined by specific requirements.

The obtained results are based on the experimental tests performed on dynamic testing systems using real manufactured products. The tests were carried out in laboratories specialized in the testing of anti-vibration and anti-seismic devices, and the devices, belonging to recognized laboratories or manufacturing companies such as SISMALAB (Italy), EUCENTRE (Italy), DIS (Reno, USA), ALGA (Italy), and ICECON (Romania).

Based on the researches and the results presented in this paper, were designed and manufactured the elastomeric anti-seismic devices used for a viaduct with a length of $200 \mathrm{~m}$, on the "Transilvania" Motorway, constructed by the Bechtel company (USA), in Romania.

The experimental tests performed in order to determine the hysterical loop families were conducted within the ICECON Romanian Research Institute for Construction Equipment and Technology - ICECON, using both anti-vibration and anti-seismic devices.

The assessment system using specialized tests was based on the Romanian Patent No. 80484/1982 "Stand for the measurement of the mechanical characteristics of rubber", whose owner is the author of this paper.

Funding: This research received no external funding.

Conflicts of Interest: The authors declare no conflict of interest.

\section{References}

1. Dobrescu, C. The Rheological Behaviour of Stabilized Bioactive Soils During the Vibration Compaction Process for Road Structures. In Proceedings of the 22th International Congress on Sound and Vibration, Florence, Italy, 12-16 July 2015.

2. Mitu, A.M.; Sireteanu, T.; Ghita, G. Passive and semi-active bracing systems for seismic protection: A comparative study. Rom. J. Acoust. Vib. 2015, 12, 49-56.

3. Sireteanu, T. Smart suspension systems. Rom. J. Acoust. Vib. 2016, 13, 2. 
4. Conveney, V.V.; Johnson, D.E.; Kulak, R.F. Modelling the Free Oscillation of Building Suported on High Damping Rubber Isolators. In Proceedings of the Seismic Engineering 2000, The 2000 ASME Pressure Vessels and Piping Conference, Seattle, WA, USA, 23-27 July 2000; Volume 1.

5. Lin, S.B. Theoretical and Experimental Study of High Damping Rubber Bearing and Lead Extrusion Damper. Master's Thesis, Feng Chia University, Taichung, Taiwan, 2000.

6. Chen, B.J.; Lin, S.B.; Tsai, C.S. Theoretical and Experimental Study of High Damping Rubber Bearings. In Proceedings of the Seismic Engineering 2001, The 2000 ASME Pressure Vessels and Piping Conference, Seattle, WA, USA, 23-27 July; Volume 2.

7. Dolce, M.; Ponzo, F.C.; Di Cesare, A.; Arlego, G. Progetto di Edifici con Isolamento Sismico; IUSS Press: Pavia, Italy, 2010.

8. Marioni, A. Sisitemi di Isplamento Sismico Innovetivi Prodotti dalla Societa ALGA. In Moderni Sistemi e Tecnologie Antisismici. Una Guida per il Progettista; 21mo Secolo: Milano, Italy, 2008.

9. Bratu, P. Vibration Transmissivity in Mechanical Systems with Rubber Elements Using Viscoelastic Models. In Proceedings of the 5th European Rheology Conference, Ljubljana, Slovenia, 6-11 September 1998.

10. Delfosse, C.G. Étude des vibrations linéaires d'un systeme mecanique complexe par méthode des mates normaux. Ann. l'ITBTP 1976, 336, 122-136.

11. Giacchetti, R. Fondamente di Dinamica delle Strutture e di Ingineria Sismica; EPC Libri: Roma, Italy, 2004.

12. Bratu, P. Analyze Insulator Rubber Elements Subjected to Actual Dynamic Regime. In Proceedings of the 9th International Congress Sound and Vibration, Orlando, FL, USA, 8-11 July 2002.

13. Bratu, P. Experimental evaluation of the antivibrating damping capacity in case of elastomers used for tram railway supportins. Mater. Plast. 2009, 46, 127-130.

14. Bratu, P. Rheological model of the neopren elements used for base isolation against seismic actions. Mater. Plast. 2009, 46, 288-294.

15. Jennings, P. Equivalent viscous damping for yelding structures. J. Eng. Mech. Division 1968, 94, $103-116$.

16. Rao, M. Mechanical Vibrations; Addison-Wesley Pub. Co.: Boston, MA, USA, 2011.

17. Sireteanu, T.; Giuclea, M.; Mitu, A.M. An analitical approach for approximation of exxperimental hysteretic by Bouc.-Wen model. Procc. Rom. Acad. Ser. A 2009, 10, 4354.

18. Sireteanu, T.; Giuclea, M.; Mitu, A.M. Identification of an extended Bouc.-Wen model with application to seismic protection through hysteretic devices. Comput. Mech. 2010, 45, 5. [CrossRef]

19. Le Tallec, P. Introduction à la Dynamique des Structures; Cépaduès: Toulouse, France, 2000.

20. Trigili, G. Introduzione alla Dinamica delle Strutture e Spettri di Progeto; Laccorio: Palermo, Italy, 2010.

21. Dolce, M.; Mortelli, A.; Panza, G. Proteggersi dal Terremoto; 21/mo Secolo: Milano, Italy, 2004.

22. Mortelli, A.; Sannino, U.; Parducci, A.; Braga, F. Moderni Sistemi e Tecnologie Antisismici Una Guida per il Progettista; 21mo Secolo: Milano, Italy, 2008.

23. Radeș, M. Mechanical Vibrationes; Ed. Printech: București, Romania, 2006.

24. Johnson, E.A.; Ramallo, J.C.; Spencer, B.F., Jr.; Sain, M.K. Intelligent Base Isolation Systems. In Proceedings of the 2nd World Conference on Structural Control, Kyoto, Japan, 28 June-1 July 1998.

25. Wang, Y.-P. Fundamentals of Seismic Base Isolation. In International Training Programs for Seismic Design of Building Structures; National Certer of Research on Earthquake Engineering: Taipei, Taiwan, 2016.

26. Stanescu, N.D. Vibrations of a shell with clearances, neo-Hookean stiffness, and harmonic excitations. Rom. J. Acoust. Vib. 2016, 13, 104-111.

27. Vasile, O. Active vibration control for viscoelastic damping systems under the action of inertial forces. Rom. J. Acoust. Vib. 2017, 14, 54-58.

28. Inman, D. Vibration with Control; John Wiley \& Sons Ltd.: London, UK, 2007.

29. Najari, S. Identification par Analyse Harmonique des Structures en Géniè Civil; INSA: Toulouse, France, 1981.

(C) 2019 by the author. Licensee MDPI, Basel, Switzerland. This article is an open access article distributed under the terms and conditions of the Creative Commons Attribution (CC BY) license (http://creativecommons.org/licenses/by/4.0/). 